\title{
Improving flight condition situational awareness through Human Centered Design
}

\author{
Carol Craig \\ Florida Institute of Technology, W. University Blvd. Melbourne, FL 32901 USA \\ Craig Technologies, 7195 Murrell Rd. Ste 101, Melbourne, Florida 32940 USA $\cdot 321.482 .0786$
}

\begin{abstract}
In aviation, there is currently a lack of accurate and timely situational information, specifically weather data, which is essential when dealing with the unpredictable complexities that can arise while flying. For example, weather conditions that require immediate evasive action by the flight crew, such as isolated heavy rain, micro bursts, and atmospheric turbulence, require that the flight crew receive near real-time and precise information about the type, position, and intensity of those conditions. Human factors issues arise in considering how to display the various sources of weather information to the users of that information and how to integrate this display into the existing environment. In designing weather information display systems, it is necessary to meet the demands of different users, which requires an examination of the way in which the users process and use weather information. Using Human Centered Design methodologies and concepts will result in a safer, more efficient and more intuitive solution. Specific goals of this approach include 1) Enabling better fuel planning; 2) Allowing better divert strategies; 3) Ensuring pilots, navigators, dispatchers and mission planners are referencing weather from the same sources; 4) Improving aircrew awareness of aviation hazards such as turbulence, icing, hail and convective activity; 5) Addressing inconsistent availability of hazard forecasts outside the United States Air Defense Identification Zone (ADIZ); and 6) Promoting goal driven approaches versus event driven (prediction).
\end{abstract}

Keywords: Human Centered Design, Situational Awareness, Aviation, Weather

\section{Introduction}

Situational awareness is recognized as a critical, yet often elusive, foundation for successful decisionmaking across a broad range of complex and dynamic systems, such as aviation and air traffic control (Nullmeyer, Stella, Montijo, \& Harden, 2005).

While flying, aircrew have a need for various types of information as the situation around them often changes at a moment's notice requiring immediate and appropriate information to respond safely. Real-time weather data is one of the most critical pieces of information utilized by the aircrew. Although there are currently a number of systems that provide different types of data such as Traffic Collision Avoidance System (TCAS), Enhanced Ground Proximity Warning System (EGPWS), Aircraft Communications Addressing and Reporting System (ACARS), and Weather Radar and Enhanced Vision System (EVS), these systems are not fully integrated, resulting in an increase in operator workload and a less than optimum awareness of potential hazards to flight. These multiple systems force the aircrew to sort through each system while simultaneously interfacing with ground controllers for information such as Pilot In-flight Reports (PIREPS) from other aircraft in the area and ground based weather radar. This type of information is inconsistent and subjective. Certain systems such as ACARS rely heavily on human communication and do not currently include weather reporting as an automated function. Wind direction and wind speed can be obtained at any time however; the data is 12 to 24 hours old. This weather data is typically based on predicted rather than actual data. There is no real-time, multi-altitude enroute wind information available with the exception of verbal communication from the controller, instantaneous wind speed and direction at current altitude from aircraft pitot/static systems, or through the home-base operations of the focus aircraft. Aircrew use radar as the primary means to avoid threatening 
weather, but the returns are only an indication of the precipitation intensity within that particular cell. To avoid life and aircraft threatening turbulence, the aircrew requests information and recommendations from the current controlling agency. The information provided comes from infrequent voice reports from other aircraft or inferior ground radar systems. The aircrew also requests information to understand which way other aircraft in the vicinity are deviating around weather.

This disconnected data gathering demonstrates the need for a more holistic approach to what kind of information reaches the aircraft and how it gets there. Aircrews have encountered situations in which echoes of weather behind radar identified weather are masked and diversions result in unintentional contact with storms. Controllers use antiquated weather radar systems and in some cases, both enroute and terminal controllers have little or no access to weather radar. For example, Miami, FL and Jacksonville, FL center controllers can see precipitation but have no insight as to intensity, elevation or precise location of weather phenomena. This lack of accurate or detailed weather data affects both civilian and military aircrew as military continuously fly through Florida airspace for training evolution and normal military logistic operations.

This field and area of study is not ignored and new technologies have been developed for cockpit presentation of graphic weather information, for turbulence prediction and warning, for automated airborne insitu weather reporting, and for data linking of weather information between airplanes in flight and providers and users on the ground. (Stough, Watson, \& Jarrell, 2010) The National Aeronautics and Space Administration (NASA) has developed technologies to reduce aviation weather-related accidents; however, these technologies have not been developed with a human centered design approach and fail to capture the user perspective at an optimum level. In the paper, New Technologies for Reducing Aviation Weatherrelated Accidents, the authors maintain that on-board weather radar, lightning detection systems, in situ reports from other aircraft and information from collaboration with ground weather briefers need to be combined effectively with the products delivered to the pilot via data-link. In addition, they maintain that means need to be developed to help pilots search the information sources available, identify trends and changes affecting their flight, and make timely decisions to avoid hazardous weather.

The authors' position in this paper is that without human-centered design and up-front consideration of interface and TOP (Technology, Organization and People) factors, any proposed solution is inadequate and incomplete. This paper is a position paper and not a finalized research product paper. The proposed solution has not been built or tested however, human centered design is necessary no matter what the results of a prototype analysis.

\section{Background}

According to the National Transportation Safety Board (NTSB), although the most common direct cause of accidents is human error, weather is a primary contributing factor in $23 \%$ of all aviation accidents. The total weather impact is estimated at $\$ 3$ billion over an eight year period and includes accident damage and injuries, delays and unexpected operating costs (Kulsea, 2010).

The weather factors that may affect airplane crashes are:

- Thunderstorms and convective weather

- In-flight icing

- Turbulence

- $\quad$ Ceiling and Visibility

According to National Aviation Safety Data Analysis Center (NASDAC) analysis, between 1989 and early 1997, weather phenomena played a part in accidents at the percentages listed in Table 1. (Kulsea, 2010):

Table 1. Weather impact on aviation accidents

\begin{tabular}{|c|c|c|c|}
\hline Condition & $\begin{array}{l}\text { Percentage of } \\
\text { accidents }\end{array}$ & & \\
\hline Thunderstorms & $2-4 \%$ & & \\
\hline Precipitation & $\begin{array}{l}6 \% \text { Commer- } \\
\text { cial }\end{array}$ & $\begin{array}{l}10 \% \\
\text { Gen Avia- } \\
\text { tion }\end{array}$ & $\begin{array}{l}19 \% \\
\text { Commuter }\end{array}$ \\
\hline Convective & $55 \% *$ & & \\
\hline In-flight Icing & $\begin{array}{l}3 \% \text { Commer- } \\
\text { cial }\end{array}$ & $\begin{array}{l}11 \% \\
\text { Gen Avia- } \\
\text { tion }\end{array}$ & $\begin{array}{l}6 \% \\
\text { Commuter }\end{array}$ \\
\hline
\end{tabular}

Convective hazards and weather conditions en route such as in-flight icing and turbulence can all lead to rerouting and diversions, thus increasing fuel and operations costs. In addition, lost revenues and passenger injuries are a possibility.

Turbulence-related injuries to cabin crewmembers occur much more frequently than turbulencerelated injuries to passengers, because cabin crewmembers are constantly working in the cabin. IATA, Safety Trend Evaluation, Analysis and Data Ex- 
change System (STEADES) performed a study of turbulence-related injuries to cabin crewmembers to better understand the impact from a crew perspective. Analysis revealed that from January 1st 2004 to December 31 st 2004 there were 232 reported cases of turbulence-related injuries to cabin crewmembers, and that:

- $64 \%$ of the injuries were due to cabin crewmembers not being secured during turbulence

- $44 \%$ of the injuries occurred in the galley

- $9 \%$ of these incidents resulted in serious injury to cabin crewmembers (Airbus, 2005).

In the majority of these cases, cabin crewmembers were lifted off the floor, or lost their balance, resulting in foot, ankle and back/spinal injuries.

Products and initiatives discussed in the Kulsea paper focus on forecast data versus real-time weather data. In addition, the described tool, Aviation Digital Data Service (ADDS) that would provide access to the most recent forecast weather conditions is dependent upon Internet access and therefore is not a favorable solution for in-flight data gathering for either civilian or government/military aviation. Although weather radar equipment is available for real-time detection of precipitation intensity, the quality and level of detail is sub-optimum and does not provide a complete picture for aircrew.

\subsection{Weather Radar}

It is common that the majority of commercial aircraft nowadays carry an Airborne Weather Radar system that is most often built into the aircraft nose. Airborne Weather Radar provides the pilot with a local (ahead only) weather picture in the cockpit and allows him to identify and avoid specific, undesirable weather formations. According to www.radartutorial.eu, published by Christian Wolff, a maximum range of $180 \mathrm{Nm}$ is common although the commonly used range (as selected by pilots) would normally be in the 30 to $80 \mathrm{Nm}$ range. The ARINC 708 is the primary weather radar system in most commercial aircraft and uses an airborne pulseDoppler radar. DOD logistics aircraft usually are delivered with systems similar to their commercial counterparts. Tactical aircraft do not have the luxury of weather radar as their systems are optimized for mission effectiveness.

\subsection{Class 2 Navigation Area}

Class 2 navigation areas are those areas outside traditional radar coverage. In these areas, there is no positive control of aircraft. Instead, the aircrew fly a prescribed route and report their position to an agency using HF radios. Traffic or weather deconfliction is not provided. There are procedures that all aircrew follow if enroute weather forces a deviation from the filed flight path. There is no sharing of weather information between aircraft. Aircrews have only the preflight weather predictions (satellite images from hours before) and their own onboard weather radar which measures only precipitation. Class 2 nav areas are all open ocean routes (trans atlantic/pacific or Caribbean to northeast (NYC etc.). Military aircraft face even more obstacles as diplomatic clearances and international law often limit flight routings and diversion options in the uncontrolled airspace.

\subsection{Interim solution inadequacies}

In addition to ADDS, there are other Internetbased weather prediction solutions that provide nearreal time data including satellite data.

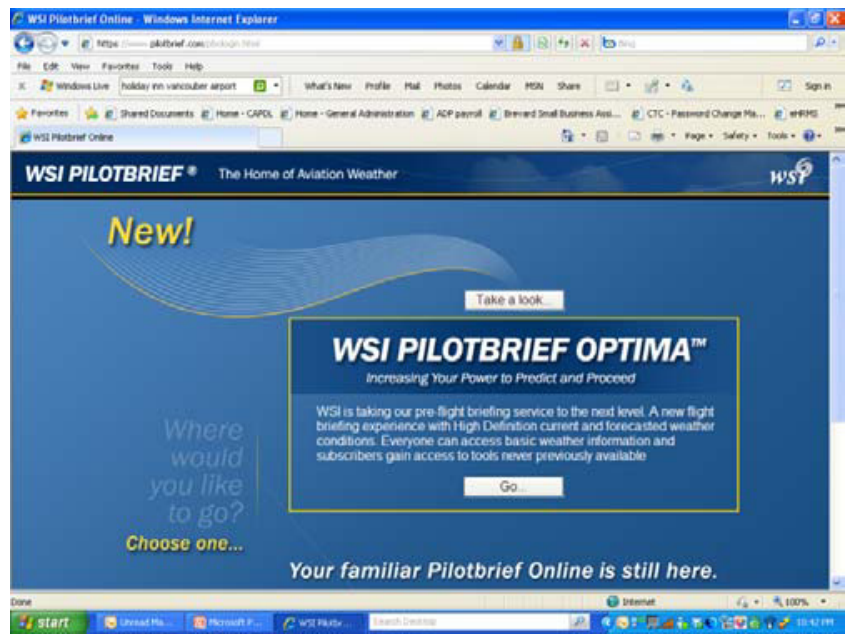

Figure 2. WSI PilotBrief Optima Portal

A press release out of Andover, MA (PRWEB) on May 03, 2011 described the Weather Services International (WSI) pilot briefing tool as a new, dramatically enhanced, on-line version of the industryleading WSI Pilotbrief ${ }^{\circledR}$ service. Pilots have the ability to receive a clear and actionable view of potential weather impacts and optimize their flight plans based on the real-time weather data. The bene- 
fits are described as including quicker aircraft turnaround times and improved mission planning and safety, among others. Similar to other planning tools, WSI is not used or recommended for in-flight weather data awareness and is dependent on internet connectivity.

\subsection{Fuel planning considerations}

Lack of knowledge of real time winds can mean fuel planning deviations and carrying extra fuel on an aircraft is an unnecessary expense. Accurate flight planning is paramount for the success of any mission.

A flight plan includes the route the crew will fly and specifies altitudes and speeds. It also provides calculations for how much fuel the airplane will use and the additional fuel it will need to carry to meet various requirements for safety. By varying the route (i.e., ground track), altitudes, speeds, and amount of departure fuel, an effective flight plan can reduce fuel costs, time-based costs, overflight costs, and lost revenue from payload that can't be carried. For military cargo aircraft, this variance of route reduces critical capabilities. These variations are subject to airplane performance, weather, allowed route and altitude structure, schedule constraints, and operational constraints.

The best route to fly depends on the actual conditions for each flight. These include the forecast upper air winds and temperatures, the amount of payload, and the time-based costs that day. The time-based costs are especially dynamic, driven by the value of the payload and the schedule and operational constraints for the crew and the airplane. Winds can have a significant impact on the optimal route and can create a significance variance between optimal and great circle "direct" route. Flight planning systems use wind forecasts from the U.S. National Weather Service and U.K. Meteorological Office, updated every one to six hours, to include the winds in every flight plan calculation. The military has dedicated weather reporting agencies, such as the Air Force Weather Agency (AFWA). The AFWA combines conventional data from civilian sources with military satellites and reporting stations to provide a realtime, integrated environmental database (Boeing, 2009).

During the Jet Fuel Asia Summit 2009, Lufthansa Consulting (2009) demonstrated the potential savings due to minimized carriage of extra fuel. This summit was tailor-made to address some of the hottest issues/main concerns in the world/Asian aviation in- dustry and jet fuel market (Rapp, 2009). The Turbulence Joint Safety Analysis Team (JSAT) recommended the below intervention for responding to the fact that turbulence has caused more serious injuries to passengers than any other class of accident:

"Improving weather information for pre-flight and in-flight turbulence avoidance decisions entails implementation of several steps. Agencies must either develop or subscribe to a comprehensive meteorological program that provides turbulence charting and alerting. The quantity and timeliness of pilot reports (PIREPS) available to pilots, dispatchers and forecasters must be improved to pinpoint location and severity of turbulence. In-flight turbulence detection capabilities are needed; turbulence forecasting must improve; and the overall utility of turbulence products for decision makers must improve."

Although not a complete solution, Honeywell developed IntuVue ${ }^{\mathrm{tm}}$, the only automatic radar able to produce a full 3-D display of weather, and the only radar with multiple options for analyzing and dissecting the weather vertically and horizontally. Using these analysis features, pilots have demonstrated a 37 percent improvement in weather decision making choosing the safest, most efficient route - by either rerouting, changing altitudes or staying on course. By improving the pilots' ability to make a more informed decision, and incorporating the latest technology for turbulence detection, IntuVue ${ }^{\mathrm{tm}}$ has shown a reduction in turbulence-related incidents by more than 45 percent, as compared to conventional radars flying on the same routes during similar times of day (Honeywell, 2011).

\section{Commercial Aviation Weather Concerns}

Through interviews, research and knowledge of the aviation industry specifically piloting, flight deck design and avionics systems, the authors believe that there is a need for a system that provides real-time integrated weather information in-flight to both commercial and military aircraft. The following description of current operations was provided during an aircrew interview: Turbulence prevention begins with comprehensive preflight planning and continues while airborne. Defenses at aircrew disposal include:

1) Preflight: Release, Dispatch, Off-Going Flight Crews, Weather Services International (WSI) Pilot Brief, Flight Operations Manual (FOM) guidance, Crew Briefings, etc. 
2) Airborne: PIREPS, Air Traffic Control (ATC), Weather Radar, Navigation Displays, Quick Reference Handbook (QRH), TCAS.

Communication and coordination among Crewmembers is a critical component of an effective response to turbulence or a threat of turbulence. Effective communication starts with the preflight briefing discussing potential turbulence threats for each leg and continues throughout the flight. The aircrews have a relatively good idea of what lies in the flight path ahead and are able to provide preliminary information to the In-flight Crew. The In-flight Crew communicates cabin conditions to the flight deck compensating for the known disparity between the ride conditions in the back of the aircraft versus the conditions in the front.

\subsection{Industry concerns}

Current radar in most aircraft display only intensity of precipitation. Much like a Doppler radar display, the information is color-coded with intensity as the differential. If there is no precipitation (return from the radar), there is no display and therefore no indication of any weather phenomena in the flight path. While cumulus nimbus and towering cumulus usually have precipitation associated with their formation, there are often instances where the cell is developing quickly and has no water component yet, resulting in no display to the aircrew. This is not as much of a problem in daytime/VFR conditions, but at night, the cell is masked and unseen. There is no inflight predictive turbulence modeling for commercial or large military aircraft. There are preflight predictions using weather reporting and interpretive wind shearing models. There are also pilot in-flight reports (PIREPS) that are subjective and not consistent. Sharing of information from preceding aircraft, real-time predictive modeling using current satellite / radar imaging and/or a turbulence function to the current radar is needed. In-flight turbulence is the number one cause of injury and aircraft damage in commercial aviation.

\subsection{Probability of Incidence}

Considering Reason's Swiss cheese model (Figure 3 ), the authors propose that weather is one of the most important holes in this model. In fact, weather combined with many other variables, especially the number of aircraft, has become a crucial issue.

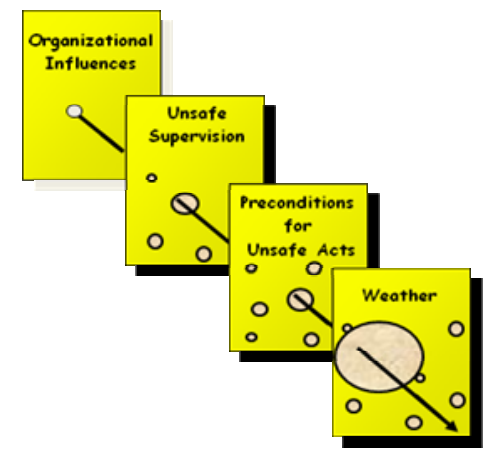

Figure 3. Reason's Swiss Cheese Model with Weather

If weather concerns can be reduced and risk mitigated with real-time insight, safety would be further improved within the entire air traffic control environment for both military and civilian entities. Already considered Ultra Safe because of worldwide regulation standardization and as a rich industry with the ability to add innovations, and with its maturity level, weather data would only further reduce the chance of accidents and improve safety and efficiency

\section{Human Centered Design Considerations}

\subsection{Complexity and the Chaos Theory}

To illustrate the complexity theory as it relates to this subject, it is first necessary to define the complex system being analyzed. Aviation, in the most general sense of the word, is a System of Systems (SoS) with the two primary (high level) systems being civil aviation and military aviation. Civil aviation is the overarching term used for all non-military aviation. It includes both General Aviation (GA) and Commercial Aviation (CA). Commercial aviation includes individual scheduled flights for the purpose of carrying passengers and/or cargo. Part 121 is the FAA term for scheduled air carrier operations (passengers). The complexity type of aviation and specifically the Part 121 flight is one of Chaos. Chaos theory has several behavioral characteristics such as 1) the system changes over time; 2) the system does not repeat itself; 3) the system can have simple causes; 4) the system is sensitive to initial conditions; 5) the system's chaotic behavior is not random; and 6) the output of the system is used as the input in the next calculation (Valle, 2000). Examples of how Part 121 flight systems meet the criteria are as follows: 1) A single flight changes over time because 
of external factors such as additional air traffic, new traffic patterns, weather, updated notice to airman (NOTAMS); 2) no single Part 121 flight is like another, even the same scheduled daily flight; 3 ) the fundamental goal of a Part 121 flight is to take off, fly from point A to point B and land; 4) initial conditions whether related to aircrew, aircraft or environment can drastically change the outcome of a flight; 5) A Part 121 flight is not a random event but rather is a well planned and scheduled event; 6) Each flight has the ability to affect another flight whether it involves ensuring the subsequent flight leaves on time (subsequent being a flight that utilizes the same gate or resources or is somehow affected by the previous) or simply alters an aspect of the follow flights as a function of time.

Chaos is supported for representation of airspace today through several steps. First, the number of airplanes in the sky and more specifically near big airports never stop to increases to the point that now the ATM system is operating at 150 percent capacity (all current airspaces were designed in the 1950s); in addition, FAA forecasts that traffic will increase to $250 \%$ within the next two decades (Swenson, Barhydt, \& Landis, 2006). This over capacity creates chaos in the mathematical sense, and this is not a metaphor. Second, the variety of aircraft is also increasing to the point that it will be difficult to manage mixed traffic of commercial aircraft, corporate jets, drones and many kinds of personal aircraft. The airspace needs to be re-thought globally, and not only locally as it was done in the past. Linear approximations are no longer working as they were when the number of airplanes was easily manageable by people such as air traffic controllers. The lack of capacity consideration leads to delays that are more and more unpredictable. (Donohue \& Shaver, 2008).

Today, everything is financially measured in the short-term, and this is precisely why we run into chaotic problems. Trying to fix local issues without addressing the global issues provides a low cost solution initially but results in paying the price eventually with exponential results. It is crucial that we address the global issue of ATM chaos because of the concerns previously described.

\subsection{Using Complexity}

The complexity of commercial and military aviation provides a useful advantage to the development of the proposed system. Viewing each aircraft as a mini weather station connects the flights and takes advantage of shared data gathering. Although capitalizing on the complexity of the various aircraft will result in complete weather data coverage, it is important to view the actual real-time weather data system as a reduced complex system because of the proposed independence between the existing cockpit system and the solution. Any proposed solution should be a well known tool that would be used intuitively by aircrew because of its familiarity. Such a solution would need to be implemented in a larger framework such as NextGen and the FAA and is global in nature, requiring national and international integration. Added complexity includes the modified scan area and new procedures to incorporate the weather data. Some verbal communication would be replaced by scanning the weather system but additional functionality would mean additional complexity and the requirement for training.

\subsection{Human-Centered Design Methodology}

Using the Artifact, User, Task, Organization, and Situation (AUTOS) pyramid to set up a weather detection safety model that uses real-time sampling of weather will help ensure the resultant system is developed with a human-centered design model (Boy, 2011). The AUTOS pyramid is a framework that helps rationalize human-centered design and engineering.

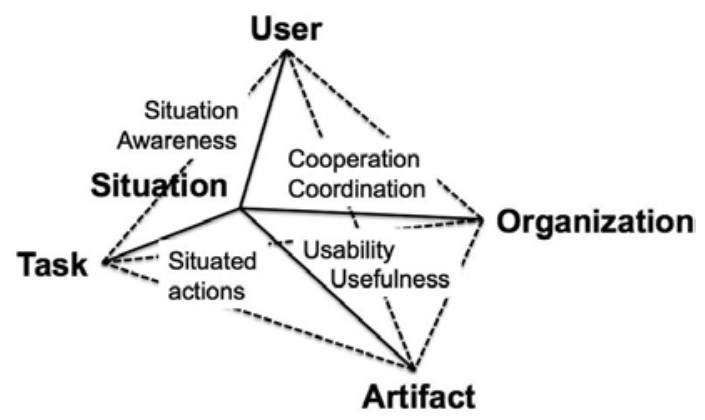

Figure 4. AUTOS Pyramid

Artifacts may be aircraft or consumer electronics systems, devices, and parts, for example. Users may be novices, experienced personnel or experts, coming from and evolving in various cultures. They may be tired, stressed, making errors, old or young, as well as in very good shape and mood. Tasks vary from handling quality control, flight management, managing a passenger cabin, repairing, designing, supplying or managing a team or an organization. Each task 
involves one or several cognitive functions that related users must learn and use.

Within the safety model of the system, considering all the barriers, the prediction of accurate weather helps keep the situation in the prevention mode, avoiding the need for recovery or mitigation. The artifact-user-task portion of the triangle can be used to define an incremental approach to design that is similar to the iterative and spiral models for software development and will be support the development of a visual weather data tool for aircrew.

The organizational environment includes all team players who can be described as "agents," whether humans or machines, interacting with the user who performs the task using the artifact. It introduces three additional edges: social issues (U-O); role and job analyses (T-O); emergence and evolution (A-O). The AU TOS framework (Figure 4) is an extension of the AU TO tetrahedron that introduces a new dimension, the "Situation," which was implicitly included in the "Organizational environment." The new edges are usability/usefulness (A-S), situation awareness (U-S), situated actions (T-S), and cooperation/coordination (O-S). To fully define the parameters or problem space relating to lack of real-time weather data using this framework, it is important to deconstruct the overall systems that make up aviation in general.

\subsection{Aviation System of Systems}

Aviation has been described as a system of systems by many researchers. Maier (1998) characterized a "system of systems" as possessing five basic traits: operational independence of elements; managerial independence of elements; evolutionary development; emergent behavior; a geographical distribution of elements. In the context of aviation, these systems have distinct operational independence (aircraft operations; maintenance; air traffic management/control) and each of these aspects has managerial independence (they are offered by independent companies, national providers, and autonomous military aviation units); however, they are bound by a set of common operating principles and international regulations for design and operation. All aspects of aviation encompass technical, human and organizational aspects. It is a socio technical system of systems encompassing critical human factors considerations such as usability, training, design, maintenance, safety, procedures, communications, workload and automation.
The aviation system of systems is a legacy system that has evolved over the past century without the benefit of design. The components within the system interact with their environment but also need boundaries in order to exist. For example, civil airlines operate into a wide range of airports (none of which they own), aircraft maintenance is often provided by third parties, aircraft ramp servicing is almost invariably provided by a range of external suppliers and air traffic management/air traffic control (ATC) is provided by the air traffic service providers from the countries into which they either operate or overfly. In the operation of civil aircraft, there are a great number of inter- and intra-organizational boundaries that information and resources must cross in this system of systems. Military aviation has an increased complexity within its system of systems because it not only interacts with the same organizations and boundaries but must interact with its own military inter- and intra-organizational boundaries and requirements. For example, tactical aircraft are incompatible with civilian approach systems and are dependent on visual flight rules (VFR).

Weather is defined as a natural system of systems and the intersection of the weather SoS and the Aviation SoS is the area of concern and focus for developing a solution. The proposed solution applies to the situation when weather directly affects aviation separately each system is extremely complex - combined, the complexity increases exponentially because of the emergent properties that are tied to the interaction of both systems

\subsection{Proposed Goals}

After deconstruction of the complex system of systems, defining the high-level goals and purposes will help ensure the design is truly human centered. Safety and human factors are often considered too late in system development to have adequate impact on the system design. The goals of the proposed system are as follows:

* To ensure pilots and dispatchers are referencing weather from the same sources

* To improve pilot awareness of forecast aviation hazards such as turbulence

* To address inconsistent availability of hazard forecasts outside the US

* To enable better fuel planning

* To allow better divert strategies

* To promote goal driven approaches versus event driven (prediction) 


\section{Prototype Development}

\subsection{Modeling Methodology}

It is difficult to develop a "flying in inclement weather" model from an observation perspective however; extensive interviewing and scenario/data capturing techniques can result in an accurate model of the overall situation. Because of the complexity of the system of systems, it is important to identify boundaries of the existing system and the situation. These boundaries should be established as a means to condense the weather situation model. Because of the importance of consideration of situational awareness and the diversity of experience within airlines and aircrew, mental models within the overall situation model should be considered. Once these models are developed, assessment of the impact of attention sharing or distraction on mental representations should be included during the participatory design phase of the process.

\subsection{Participatory Design}

A lifecycle process that combines a standard iterative process, rapid prototyping and an "operator directed process" that inserts the development of training material would result in a user centric design process and final product (Vakil \& Hansman, 2002). Previous incorporation of weather data has been void of user involvement, user training and user understanding. The majority of civil aviation aircrew are unfamiliar with the radar in various aircraft and do not fully understand wave forms, radar return physics nor 2D visual displays. Some former military aviators are able to better understand the distances and implications based on beam width but generally, aircrew do not have a good understanding of the radar data at a detailed level. Providing a solution using familiar technology developing visual displays that promote recognition versus recall would be a vast improvement from the current radar. A 3-D depiction can be modeled during the prototype phase to ensure the solution is feasible and acceptable to the user community.

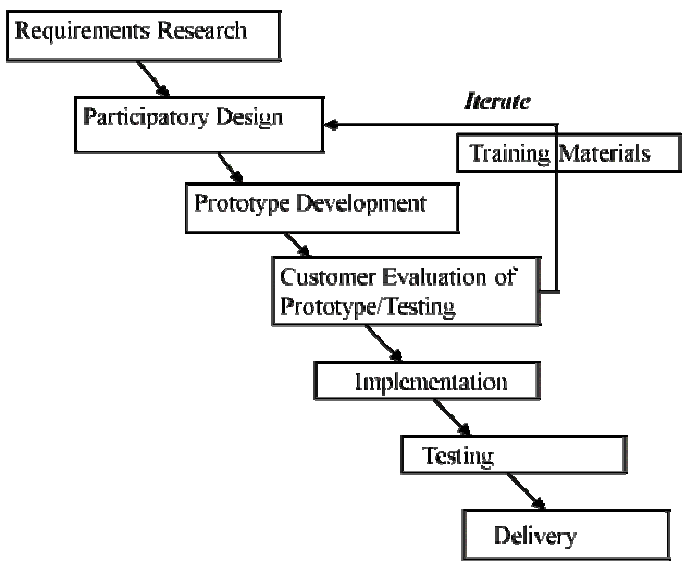

Figure 5. Proposed Modified Software Life Cycle Process

\section{Proposed Solution}

Any proposed system should be considered as a visual extension of the existing cockpit system providing an accurate, timely and situationally relevant real time weather depiction which can be simulated virtually for training and implementation. Specific weather data related to conditions that require immediate evasive action by the flight crew, such as isolated heavy rain, microbursts, and atmospheric turbulence includes information about the type, position, and intensity of those conditions. A real-time 3D weather data display would act as a virtual camera for displaying all types of weather phenomena so aircrew can make real-time decisions regarding changes to flights paths to accommodate conditions. In addition to software changes, external weather data sampling hardware will be necessary to gather real-time information about each aircraft and share that information as if each airplane were a mini weather station. Using an independent piece of equipment would allow easier integration into the current cockpit system and real estate. As Alan Kay stated, "The best way to predict the future is to invent it." The authors' position is that the best way to invent the safest future for aviation is to have the most accurate depiction of the current situation and the near future situation. This can be done with improved weather situational awareness and real-time sampling of weather. Creating mini-weather stations from each aircraft in the sky would provide an abundance of real-time weather data. 


\subsection{Adaptation}

One of the goals of the real-time weather data system should be the reduction of reactive responses with an "event approach" and the promotion of an "anticipation approach." This type of proactive approach will reduce the need to react to adverse weather events, especially those that might be occurring simultaneously with other emergency events.

When considering the adaptation requirements, 1) intuition (ensure flight crew interacts easily), 2) information (ensure useful data can be captured) and 3) return on investment (ensure that the solution is worth the changes to aircraft and cockpit) will need to be carefully evaluated to determine how many levels of adaptation are necessary and the detail required for each. Three primary considerations that must be evaluated are: 1) Addition of a new system/software as an extension of the current cockpit system and physical integration of iPad mounts; 2) Addition of external data collection hardware on each aircraft; 3) Procedures for scan, role/responsibility and dissemination of weather data (replacing the calls to the controller).

Individual airlines will need to integrate the solution based on FAA approved methods and would be responsible for all training and procedural integration. Because of the unique requirements of each organizational fleet whether civilian or military, this integration will differ greatly across each organization and will need to be led by knowledgeable experts.

\section{Conclusion}

In conclusion, findings and feedback from this project suggest that further development of the full design is desired by both researchers and users. Using a holistic and systematic approach with a HumanCentered design strategy addresses the human factors issues that arise when considering how to display various sources of real-time weather information to the users of that information and how to integrate the display into the existing environments. In designing weather information display systems, it is necessary to meet the demands of different users, which requires an examination of the way in which users process and use weather information. Using HumanCentered Design methodologies and concepts will result in a safer, more efficient and more intuitive solution. Preferred weather data provided by the system should include wind speed, wind direction, storm patterns, turbulence levels, and other data affecting flight with specially designed groupware for the pilot community with tailored cognitively unambiguous technologies. Providing data from multiple sources and systems into one consolidated display results in an integrated all-inclusive system with the highest level of situational awareness and with an advanced prediction factor to be determined.

\section{References}

[1] Airbus Flight Operations Briefing Notes / Turbulence Threat Awareness (2005). Retrieved from http://www.airbus.com/fileadmin/media_gallery/files/safety_li brary_items/AirbusSafetyLib_FLT_OPS-CAB_OPSSEQ10.pdf

[2] Altus, S. (2009). Effective Flight Plans Can Help Airlines Economize. Boeing Aero, QTR_03.09. Retrieved from http://www.boeing.com/commercial/aeromagazine/articles/qtr 03 09/article_08_1.html

[3] Boy, G. (2011). The Handbook of Human-Machine Interaction: A Human-Centered Design Approach. Ashgate, U.K.

[4] Donohue, G. \& Shaver, R. (2008). Terminal Chaos: Why U.S. Air Travel is Broken and How to Fix it. Reston, VA: American Institute of Aeronautics and Astronautics, Inc.

[5] Kulsea, Gloria (2002). Weather and Aviation: How Does Weather Affect the Safety and Operations of Airports and Aviation, and How Does FAA Work to Manage Weatherrelated Effects? Retrieved from http://climate.dot.gov/documents/workshop1002/kulesa.pdf

[6] Nullmeyer, R.T., Stella, D., Montijo, G.A., \& Harden, S.W. (2005). Human factors in Air Force flight mishaps: Implications for change. Proceedings from 27th Annual Interservice/Industry Training, Simulation, and Education Conference (paper no. 2260). Arlington, VA: National Training Systems Association.

[7] Rapp, T. (2009). Cost Savings Through Operational Excellence In The Environment of Volatile Fuel Prices. [PowerPoint slides]. Retrieved from http://www.lhconsulting.com/fileadmin/images/www.lhconsul ting.com/downloads/speeches/090304_Cost_Saving_Fuel_Mg t_SIN_FINAL.pdf.

[8] Stough, H., Watson, J., \& Jarrell, M. (2006). New Technologies for Reducing Aviation Weather-Related Accidents. Retrieved from

http://ntrs.nasa.gov/archive/nasa/casi.ntrs.nasa.gov/200600483 02_2006250471.pdf

[9] Swenson, H., Barhydt, R., \& Landis, M. (2006). Next generation air transportation system air traffic managementairspace project [PowerPoint slides]. Retrieved from $\mathrm{http}: / /$ ntrs.nasa.gov/search.jsp? $\mathrm{Ntx}=$ mode $\% 20$ matchallany $\& \mathrm{Nt}$ $\mathrm{k}=\mathrm{All} \& \mathrm{Ns}=$ Loaded-

Date $\mid 1 \& \mathrm{~N}=4294574760 \& \mathrm{Ntt}=\mathrm{Next} \% 20$ generation $\% 20$ air $\% 20$ transportation $\% 20$ system $\% 20$ air $\% 20$ traffic $\% 20$ managementairspace $\% 20$ project

[10] Vakil, S.,. \& Hansman, R. (2002). Approaches to mitigating complexity-driven issues in commercial autoflight systems., 143. Retrieved from http://web.mit.edu/aeroastro/www/people/rjhans/docs/RESS2 965.pdf 